\title{
AN ANALYSIS OF STATISTICAL TEXTS THROUGH THE MEANING ELEMENTS OF GODINO
}

Teresita E Terán $^{1}$, Augusto Nascimbene ${ }^{1}$, and Diana Kohan ${ }^{2}$

${ }^{1}$ Facultad de Ciencias Veterinarias - Universidad Nacional de Rosario

${ }^{2}$ Facultad de Ingeniería. Universidad Nacional de Entre Ríos

teresitateran@hotmail.com

Through some research projects, studies have been carried out on the contents of essential university textbooks of Basic Statistics. Access to textbooks as "epistemological tutors of wise knowledge" is fundamental at the university level. At that level, instruction in statistics is primarily instrumental. An a priori analysis of the text allows us to identify and anticipate possible epistemological and didactic obstacles that present generalized difficulties and will generate frustrations and errors for some students. In this poster, we use the theoretical framework of Godino to present an analysis of text content that is incorporated into the integral study of the Institutional Meaning of Reference in the topic Confidence Intervals, in the Faculties of the National Universities in Argentina. 